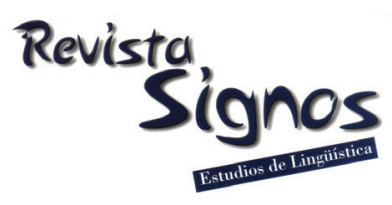

\title{
La presencia del inglés en la red de Internet en lengua española: Las ofertas de empleo en www.monster.es*
}

\author{
The presence of English on the Internet in Spanish: Job \\ advertisements in www.monster.es
}

Andreu van Hooft

RAdBoud University NijMEGEN

HOLANDA

a.v.hooft@let.ru.nl

Recibido: 5-IV-2014 / Aceptado: 22-I-2015

\section{Resumen}

En este artículo presentamos los resultados de un estudio empírico y sincrónico sobre la presencia de la lengua inglesa en un corpus de 208 ofertas de empleo publicadas en lengua española en el sitio web www.monster.es durante el mes de noviembre de 2011. El propósito de este estudio ha sido identificar la presencia de anglicismos en tanto que 'préstamos no adaptados', usados en las ofertas de empleo, determinar su presencia y cantidad y establecer hasta qué punto la presencia del inglés depende de los siguientes factores extralingüísticos: el sector de actividad económica, el rango de la ocupación anunciada (alto, intermedio o bajo) y el tipo de organización (internacional o nacional). Los resultados obtenidos indican que el porcentaje de ofertas de empleo que contienen por lo menos una palabra inglesa $(87,5 \%)$ superó significativamente el porcentaje de ofertas de empleo redactadas exclusivamente en español (12,5\%). Mediante la aplicación de las pruebas estadísticas $U$ de Mann-Whitney, Kruskal-Wallis y el coeficiente de correlación de Spearman (rS) hemos podido verificar que una mayor presencia del inglés depende del sector económico, del rango de la vacante y el carácter internacional de la empresa anunciante. Estos hallazgos corroboran las razones dadas por otros autores según las cuales las empresas internacionales pueden usar el inglés para ofrecer una imagen internacional consistente y como herramienta para seleccionar candidatos. $\mathrm{Y}$, en línea con los resultados de otros estudios de corpus y lexicográficos previos, también aquí el sector de las nuevas tecnologías de la información y la comunicación (TIC) es el que adopta la mayor cantidad de préstamos no adaptados del inglés.

Palabras Clave: Ofertas de empleo, análisis de corpus, cuantitativo, anglicismos no adaptados, Internet. 


\begin{abstract}
In this paper we present the results of a corpus-based synchronic study on the presence of English in 208 jobs ads placed on the Spanish website www.monster.es published during the month of November 2011. The aims of this study were to identify the presence of anglicisms used in job ads, determine their presence and quantity and establish to what extent the presence of English depends on the following extra linguistic factors: industry activity, job level (high, intermediate or low) and type of organization (international or domestic). The results indicate that the percentage of job ads that contained at least one English word (87.5\%) was significantly higher for the job ads written exclusively in Spanish (12.5\%). Using the $U$ de Mann-Whitney and Kruskal-Wallis test as well the Spearman (rS) correlation test, we verify the extent to which English was used, was found to depend on the industry activity, job level, and (inter)national orientation. These findings support observations in previous research: international companies can use English to provide a consistent image and can be used as a tool for selecting candidates. And, in line with the results of corpus-based studies and previous lexicographical works, this study shows that new information and communication technologies (ITC) sector uses the most non-adapted loan words from English.
\end{abstract}

Key Words: Job ads, corpus analysis, quantitative, non-adapted anglicisms, Internet.

\title{
INTRODUCCIÓN
}

La influencia y presencia del inglés en la lengua española en general y, particularmente, en el español de las profesiones es un hecho indiscutible que ha sido documentado a través de numerosos estudios y artículos aparecidos en revistas especializadas y prensa general dedicados a los anglicismos tanto en España (véase la documentada bibliografía de Rodríguez González, 2002a, 2002b) como en los países hispanoamericanos (Baumgardner, 2006). Los resultados de estos estudios han servido para realizar inventarios de préstamos no adaptados procedentes del inglés y para la creación de diccionarios de anglicismos como, por ejemplo, el Nuevo Diccionario de Anglicismos (Buades \& González, 2009) y, así, describir la presencia e influencia de la lengua inglesa en el español. No obstante, una mayoría de los estudios realizados hasta hoy en el ámbito hispánico son de tipo cualitativo y los que son cuantitativos se limitan, a menudo, a medir la presencia del inglés en la lengua española a partir de estadísticos descriptivos básicos como la cantidad de ocurrencias y proporción de palabras inglesas en corpus orales (Quilis, 1984; Gómez Capuz, 2009) y escritos. Incluso en los estudios de corpus de enfoque cuantitativo que han estudiado textos escritos en lengua española, no todos los géneros textuales han recibido la misma atención, lo que dificulta sacar conclusiones generalizables. Así y mientras la presencia de inglés en la prensa (Mighetto, 1991, 1992; Rodríguez González, 2002a; Gerding, Fuentes, Gómez \& Kotz, 2012) y la publicidad (Durán Martínez, 2000; van Hooft, 2006; Cruz, Tejedor Díez \& Cerdá, 2007; Gerritsen, Nickerson, Crijns, van Hooft, 
van Meurs, Nedertigt \& Starren, 2007) ha sido, hasta cierto punto, cuantificada, demostrada de forma empírica y explicada tanto en los países de habla hispana como en otros ámbitos lingüísticos (Piller 2003; Zenner, Speelman \& Geeraerts, 2013). No obstante, los estudios empíricos de enfoque cuantitativo que han tratado de determinar y explicar la presencia del inglés en la red de Internet en general y, en particular, las ofertas de empleo publicadas en dicha red son escasos, y en el ámbito hispano podemos afirmar que inexistentes. Es decir, que hasta el presente no existe una descripción ni cuantificación de la presencia del inglés - sea a micro nivel (la presencia voces foráneas procedentes del inglés o anglicismos) sea a macro nivel (la presencia de textos completamente redactados en inglés en el ámbito hispano)- que nos permita determinar el impacto de esta lengua en las ofertas de empelo en lengua española publicadas en Internet.

De ahí que el objetivo de este estudio descriptivo sea identificar la presencia de anglicismos usados en las ofertas de empleo publicadas www.monster.es, determinar su presencia y cantidad, y establecer hasta qué punto la presencia del inglés depende de los siguientes factores extralingüísticos: el sector de actividad económica, el rango de la ocupación anunciada (alto, intermedio o bajo) y el tipo de organización (internacional o nacional).

De lo dicho hasta ahora se desprenden las preguntas de esta investigación:

PI 1: ¿Cuál es la presencia del inglés en el portal de internet de www.monster.es?

PI 2: ¿Cuántas ofertas de empleo contienen palabras inglesas?

PI 3: ¿Depende el uso de palabras inglesas en las ofertas de empleo de www. monster.es del sector de actividad económica de la organización que anuncia la vacante?

PI 4: ¿Aparece el inglés con más frecuencia en las ofertas de empleo para ocupaciones de nivel alto o en las ofertas para ocupaciones de nivel medio o bajo?

PI 5: ¿Hasta qué punto depende la presencia del inglés en las ofertas de empleo del tipo de organización (internacional versus nacional)?

A continuación y para justificar teóricamente las preguntas de la investigación presentadas arriba, primero describiremos los antecedentes y, en segundo lugar, expondremos y discutiremos los factores lingüísticos y extralingüísticos que pueden determinar la elección lingüística, es decir, el uso de los 'anglicismos no adaptados' en este corpus. 


\section{Los antecedentes: Estudios sobre la presencia del inglés en estas ofertas de empleo en la prensa escrita e Internet}

Los primeros estudios que han detectado la presencia del inglés en las ofertas de empleo aparecidas en la prensa de países de habla no inglesa fueron los de Larson (1990) para la prensa sueca, Hilgendorf (1996) para la alemana y Taavitsainen y Pahta (2003) para la prensa finlandesa. No obstante, ninguno de estos estudios determinó cuantitativamente la presencia del inglés en dichas ofertas de empleo, dado que se trataban de estudios cualitativos, basados en evidencias empíricas limitadas. El primer estudio de enfoque empírico y cuantitativo sobre presencia del inglés en las ofertas de empleo publicadas en un ámbito lingüístico donde el inglés no es lengua oficial y en la prensa escrita, se debe a Korzilius, van Meurs y Hermans (2006), quienes determinaron que un 2,4\% de las 679 ofertas publicadas en el mes de agosto del año 2001 en el periódico neerlandés De Volkskerant fueron escritas completamente en inglés. Además y después de un análisis más detallado de las 119 ofertas que no fueron escritas completamente en inglés, estos autores establecieron que el 39\% de estas ofertas contenía una o más palabras inglesas. Por lo que se refiere al estudio de las ofertas de empleo publicadas en Internet, solo hemos encontrado un estudio que haya determinado y cuantificado la presencia del inglés en este género de texto. Se trata del análisis de un corpus de ofertas de empleo publicadas en el portal www. monsterboard.nl de van Meurs, Korzilius y Den Hollander (2006). Estos autores determinaron que un $88,5 \%$ de las ofertas de empleo del corpus estudiado contenían una o más palabras inglesas, mientras que solo un $11,5 \%$ de las ofertas fue escrito exclusivamente en neerlandés.

\section{Los factores que pueden explicar la presencia del inglés en las ofertas de empleo}

\subsection{El sector de actividad económica de la organización que anuncia la vacante}

Las áreas de actividad sensibles a la adopción de anglicismos y donde es más visible su penetración, ya sea por las innovaciones técnicas ya sea por el carácter internacional de las mismas son: la comunicación audiovisual, radio y televisión, Internet, las áreas de actividad relacionadas con la economía, las finanzas y el comercio, el mundo del deporte, la moda, el turismo, la ciencia y técnica en general y las nuevas tecnologías de la información y comunicación (TIC) (Blanco 1995; Calvi, 2000; Lázaro Carreter, 2002; Lorenzo, 1996; Rodríguez González, 2002a; van Hooft, 2006; Cruz et al., 2007; Álvarez, 2011 ). Lo mismo puede aplicarse para la publicidad en otros países de habla no inglesa (Cheshire \& Moser, 1994; Griffin, 1997). Por ejemplo y en el caso de la lengua neerlandesa, van der Sijs (1996) observó que la presencia de palabras inglesas es muy frecuente en los sectores relacionados con las TIC. Observación válida también para la prensa escrita en seis variantes geolectales del español tal y como han 
demostrado Gerding et al. (2012) en su extenso análisis de corpus. Mientras que otros estudios (para Europa, Kelly-Holmes, 2000; Piller, 2003; Ustinova, 2006; Gerritsen et al., 2007; para Asia, Haarmann, 1989; Bhatia, 2001; para Hispanoamérica, Friedrich, 2002; Alm, 2003; Baumgardner, 2006) han determinado que la lengua inglesa en tanto que lengua extranjera, se usa a menudo en campañas publicitarias del sector de la moda, cuidado personal y lujo. Sin embargo y por lo que a evidencia empírica y cuantitativa se refiere, los resultados de los análisis de corpus dedicados a la ofertas de empleo son contradictorios. Así y mientras en la investigación de Korzilius et al. (2006) no se encontraron diferencias en cuanto al uso del inglés según el sector de actividad económica, en el estudio de van Meurs et al. (2006) por lo contrario, sí se halló una mayor presencia significativa de palabras inglesas en las ofertas de empleo pertenecientes al sector de las TIC. Hallazgo, este último, que ha sido corroborado por el reciente estudio de Zenner et al. (2013), en el que también la presencia del inglés es significativamente mayor en las ofertas de empleo de empresas pertenecientes a este sector. Mientras que las organizaciones estatales, organizaciones culturales y sociales usan significativamente menos inglés que los otros sectores.

\subsection{El rango de la función ofertada}

El uso del inglés en las ofertas de empleo tendría como fin añadido atraer la atención del grupo meta hispano. Pero el uso del inglés no solo serviría para atraer la atención del lector, otro objetivo prioritario sería el de seleccionar un grupo meta muy determinado. En el sentido de que las ofertas de empleo escritas mayoritariamente o exclusivamente en inglés solo podrán ser leídas por hablantes hispanos que dominen el inglés como lengua extranjera o segunda lengua. De ahí que el uso que esta lengua tenga también puede tener como objetivo preseleccionar a los posibles candidatos, dado que, seguramente, un buen dominio de la lengua inglesa será uno de los requisitos necesarios para obtener el puesto. Estos candidatos, además de tener unas competencias profesionales determinadas para poder realizar las labores requeridas, también deberán saber inglés. En este sentido, se podría esperar que las ofertas que incluyen inglés o que incluso han sido redactadas exclusivamente en inglés estuvieran asociadas a vacantes de un nivel socioeconómico alto que, a su vez, requerirían un nivel educativo alto por parte de los candidatos potenciales. Sobre esta expectativa y hasta la fecha y en cuanto a la asociación de la presencia del inglés según el parámetro del nivel socioeconómico ofrecido y el nivel educativo exigidos en las ofertas de empleo, los resultados obtenidos por Korzilius et al. (2006) y van Meurs et al. (2006) apuntan en direcciones opuestas. De tal modo que en el primer estudio no se halló ninguna diferencia significativa en cuanto a la presencia del inglés según niveles socioeconómicos y educativos en las ofertas de empleo neerlandesas. Mientras que en el segundo estudio, sobre el impacto del inglés en las ofertas publicadas en www. monsterboard.nl, sí se observó que en las ofertas de empleo para ocupaciones de rango alto (de un nivel socioeconómico y educativo alto) usaban significativamente más palabras inglesas que las oferta de rango medio o bajo. 


\subsection{El tipo de empresa}

Una de las razones más nombradas que explicarían el uso del inglés en la publicidad es el hecho de que estas campañas forman parte de una estrategia internacional de marketing (Jain, 1989; De Mooij, 1994; Piller, 2001). En este sentido y según las agencias de publicidad, el uso del inglés se debería a la necesidad de crear una estrategia global y consistente de marketing (Gerritsen, Korzilius, van Meurs \& Gijsbers, 2000; $\mathrm{Alm}, 2003)$. Las organizaciones internacionales usan los mismos anuncios, nombres de marca y producto, o frases publicitarias en inglés y en diferentes países. Larson (1990) afirma que al usar los mismos términos ingleses en los títulos de las funciones de las vacantes anunciadas en las ofertas de empleo publicadas en diferentes países, las compañías pretenden crear una imagen de empresa consistente. Y de paso y tal como sostienen Jain $(1989,1993)$ y Walsh (1991), el uso de esta lengua supone un ahorro de los gastos de traducción y adaptación de las campañas de publicidad y ofertas de empleo a otras lenguas. Tal y como afirma Harmann (1989: 234) el inglés se usaría también porque "is found to index a social stereotype of modernization, sophistication and cosmopolitanism". Estas connotaciones vendrían explicadas, en primer lugar, por la diseminación y estatus del inglés (Görlach, 2002; Crystal 2003a, 2003b) y el prestigio social de esta lengua (Haarmann, 1989; Griffin, 1997; Friedrich, 2002). Prestigio social motivado por razones extra lingüísticas como el hecho de que, a menudo la innovación tecnológica y científica se dé en los Estados Unidos o el Reino Unido o en y entre empresas multinacionales o en y entre universidades en las que, a menudo, el inglés es la lengua franca de comunicación.

Las razones y motivos arriba expuestos según los cuales las empresas internacionales utilizarían más el inglés e incluso publiquen más ofertas de empleo únicamente en inglés, han sido corroborados para la lengua holandesa y mediante evidencia empírica en los estudios de Korzilius et al. (2006) y van Meurs et al. (2006). En ambos estudios, tanto el dedicado a las ofertas publicadas en la prensa como en www.monsterboard. $\mathrm{nl}$, las ofertas de empleo colocadas por empresas internacionales y multinacionales mostraron de forma significativa una mayor concentración de palabras inglesas que las ofertas de empleo colocadas por las empresas domésticas holandesas.

\section{Marco metodológico}

\subsection{El corpus}

La muestra analizada consistió en 208 ofertas de empleo publicadas en el portal de Internet de la filial española de Monsterboard (www.monster.es). Dicha muestra fue extraída aleatoriamente de un corpus de 800 ofertas de empleo aparecidas durante el 4 de noviembre y el 18 de noviembre de 2011 en dicho portal. El tamaño de la muestra ofrece un nivel de confianza del $90 \%$ y un error de muestreo de un $10 \%$ (Korzilius, 2000). Estas 800 ofertas de empleo fueron seleccionadas mediante la opción de búsqueda 'busca empleos' que ofrece el portal de dicha empresa, sin indicar 
la localidad, ni la categoría de trabajo y, además, sin usar otras palabras clave de búsqueda. La muestra analizada en este artículo se extrajo de este corpus de anuncios de empleo mediante la opción 'muestra aleatoria de casos' ofrecida por el programa de análisis estadístico IBM SPSS versión 21.0 (2012). En el portal de www.monster. es, las ofertas de empleo pueden ser seleccionadas de tres maneras: 1) solo con el nombre de la profesión o trabajo, el nombre de la empresa y el lugar; 2) solo con el nombre de la profesión o trabajo, el nombre de la empresa, el lugar y un breve resumen del anuncio de empleo; 3) el anuncio de empleo completo. Decidimos analizar los anuncios completos dado que son el tipo de anuncios que contiene más texto.

\subsection{La definición de los términos 'palabra', 'palabra española' y 'palabra inglesa'}

En primer lugar y para poder determinar la cantidad de palabras en inglés y castellano aparecidas en el corpus de ofertas de empleo, es necesario definir primero qué es una palabra. La definición que hemos utilizado es la que ofrece el Diccionario de la Lengua Española (2003, versión digital), "segmento del discurso unificado habitualmente por el acento, el significado y pausas potenciales inicial y final". Entendemos que cuando la Academia de la Lengua se refiere a un 'segmento del discurso' se está refiriendo a un carácter o una serie consecutiva de caracteres aparecidos entre espacios, o entre un espacio y una marca de puntuación. Si este es el caso, la definición de la Academia de la Lengua coincidirá con las definiciones de 'palabra' dadas por los diccionarios ingleses Merriam-Webster Online Dictionary (versión en línea, 2010) y Oxford Online Dictionary (versión en línea, 2010). También hemos considerado como palabras inglesas aquellos neologismos no registrados en los dos diccionarios de la lengua inglesa antes mencionados, pero cuya morfología y ortografía pueden considerarse palabras inglesas, como por ejemplo, timewear.

En segundo lugar, hemos considerado como palabras españolas todas aquellas palabras registradas en el Diccionario de la Lengua Española (versión en línea, 2003), con la excepción de los 'préstamos no adaptados' o extranjerismos incluidos en el mismo diccionario como, por ejemplo, golf catalogado como 'voz inglesa', forfait como 'voz francesa', leitmotiv como 'voz alemana'.

En tercer lugar, hemos considerado como palabras inglesas todas aquellas palabras o frases que han sido registradas en los dos diccionarios de la lengua inglesa antes mencionados, exceptuando, claro está, los extranjerismos registrados en ambos diccionarios. Además y complementando estas dos fuentes lexicográficas, también hemos consultado otros recursos lexicográficas informatizados como el British National Corpus (2011) o buscadores como Google (2011) y Ngram Viewer (2011) para determinar si la morfología, ortografía, gramática y sintaxis eran inglesas.

De esta manera hemos podido fijar la cantidad de palabras inglesas utilizadas (los casos/tokens) en el corpus de ofertas de empleo analizado. En este sentido y matizando, 
en nuestro análisis hemos considerado solamente como anglicismos las formas inglesas no adaptadas a la morfología y ortografía de la lengua castellana, es decir, los llamados también 'préstamos no adaptados'. Incluso aquellos extranjerismos ingleses o préstamos no adaptados como, por ejemplo, marketing, slip o software incluidos en el Diccionario de Lengua Española como voces inglesas, también han sido considerados como anglicismos no adaptados, como palabras inglesas, en nuestro estudio. Por lo contrario, las formas adaptadas a la morfología y ortografía de la lengua española, los llamados 'préstamos adaptados' procedentes del inglés y registrados en el Diccionario de la Lengua Española, como 'voces del inglés', por ejemplo, 'jungla', 'líder' o 'digitalizar', no han sido consideradas como palabras inglesas sino ya como parte del léxico de la lengua española.

En cuarto lugar y junto a las palabras inglesas y españolas, hemos distinguido una tercera categoría de palabras, las palabras híbridas. Un tipo de palabra o grupo de palabras en las que en un mismo segmento del discurso se combina una parte en inglés y otra en castellano. Este es el caso de muchas direcciones de portales de Internet en las que se suele integrar una parte en inglés, las siglas [www] (world wide web) y otra parte en español que incluye tanto los nombres completos o los acrónimos o las siglas de la empresa o institución como las abreviaturas [es] (España) o [gob] (gobierno). Sirvan las siguientes direcciones como botón de muestra: www.lacentrallechera.es, www.educacion.gob.es/ y www.rae.es. Mediante la distinción de esta tercera categoría seguimos aquí el mismo criterio morfológico aplicado por van Meurs et al. (2006) y van Hooft (2006), en su análisis de un corpus de anuncios aparecidos en la edición española de la revista Elle. En este sentido y en este estudio discrepamos de la elección realizada por Gerritsen et al. (2007), en cuyo estudio contabilizaron las direcciones de los portales de internet como palabras inglesas.

Finalmente, todas aquellas palabras o préstamos no adaptados procedentes de otros idiomas como el francés, catalán, alemán o italiano han sido incluidas en una cuarta categoría.

\subsection{La determinación del sectory la clasificación deocupaciones}

Hemos seguido la clasificación de los sectores de actividad económica estipulados por la Comisión Europea (2006) para determinar a qué sector pertenecen las empresas anunciantes. Para determinar a qué rango pertenecen las vacantes anunciadas en las diferentes ofertas de empleo, hemos consultado las clasificaciones de ocupaciones ofrecidas por el Instituto Nacional de Estadística (INE, 2011), el Instituto de Estadística Neerlandés (CBS, 2010) y la Organización Internacional del Trabajo (Hoffmann \& Scott, 2011). Después de contrastar dichas clasificaciones y comprobar que comparten las nueve categorías básicas de ocupaciones profesionales, hemos reducido estas nueve categorías a tres de acuerdo con el nivel de competencias profesionales indicado en la cuarta columna de la tabla de clasificaciones presentada por Hoffmann y Scott 
(2011). Luego, hemos determinado que las ocupaciones del rango más alto lo ocupan, por ejemplo, cargos de dirección de empresa y administraciones públicas técnicos y profesionales científicos con un nivel de competencia laboral 4 y 3. El rango intermedio incluye, entre otros, a los técnicos profesionales de apoyo y empleados de tipo administrativo con un nivel de competencia 2. Y por último, el rango bajo se corresponde con los trabajadores no cualificados o ocupaciones elementales con un nivel de competencia 1.

\subsection{La determinación del tipo organización: ¿Internacional o nacional?}

Para determinar si una empresa es nacional o internacional (incluidas aquí las multinacionales) hemos utilizado los siguientes criterios, establecidos por van Meurs et al. (2006):

1. la empresa realiza actividades en el extranjero o tiene clientes en más de un país;

2. las filiales españolas y las filiales en otros países son nombradas en la oferta de empleo o en el portal de la empresa u organización;

3. la empresa u organización forma parte de una empresa u organización española que tiene filiales en otros países, según la información dada en la oferta de empleo o en el portal de internet de la empresa;

4. las direcciones de los portales de las filiales situadas fuera de España fueron localizadas cambiando la extensión 'es' (el código o abreviatura para España) por '.com' o ‘.org', o la extensión de otros países (por ejemplo '.nl', Países Bajos);

5. la oferta de empleo especificaba de forma explícita que la empresa era una empresa internacional o multinacional.

Si la organización no cumplía con estos requisitos y el portal de Internet de la empresa u organización mencionaba que solo estaba establecida en España hemos considerado estas empresas como empresas nacionales. Cuando no pudimos encontrar suficiente información sobre cualquiera de los puntos arriba expuestos - por ejemplo, el nombre de la organización no aparecía en la oferta de empleo-, decidimos que estas empresas tenían un estatus desconocido al respecto. Por último, no incluimos en nuestro estudio aquellas ofertas de empleo de multinacionales colocadas por otras empresas intermediarias en nombre de dichas multinacionales en el corpus objeto de análisis.

\subsection{Análisis estadísticos}

Las frecuencias, los promedios, desviaciones típicas y medianas han sido calculadas con la ayuda del programa de análisis estadístico de IBM SPSS versión 
21.0. (2012). Mediante la aplicación de la prueba estadística de Kolmogorov-Smirnov hemos podido establecer que la variable dependiente, es decir, la cantidad de palabras inglesas, no estaba normalmente distribuida a través de la muestra analizada. Por esta razón y para determinar si hay diferencias estadísticas significantes, hemos utilizado pruebas estadísticas no paramétricas. Así y para determinar la significancia de la diferencias entre dos grupos independientes utilizamos la prueba estadística de la U de Mann-Whitney, y para el análisis de tres o más grupos la prueba estadística de Kruskal-Wallis. Por último y para comprobar si existen relaciones entre las variables investigadas, hemos usado el coeficiente de correlación de Spearman $\left(\mathrm{r}_{\mathrm{s}}\right)$.

\section{Resultados}

En esta sección presentamos los resultados de nuestro análisis del corpus de oferta de empleo de www.monster.es.

\subsection{Presencia del inglés en las ofertas de empleo de www. monster.es}

Según se desprende del análisis de la muestra de 208 ofertas de empleo, del total de 51.832 palabras usadas, el 56,9\%, es decir, 29.496 palabras eran españolas; 20.628 palabras, el 39,8\% inglesas; el 1,7\% (880 palabras) eran formas híbridas y, finalmente, 828 palabras procedían de otros idiomas lo que representa el 1,6\% de la muestra.

Por un lado y del total de 208 ofertas de empleo, 23 de ellas han sido redactadas completamente en inglés lo que representa el 11,1\% de la muestra; y, por otro lado, 26 ofertas exclusivamente en lengua española, es decir, el 12,5\% de la muestra. El resto de ofertas, $159(76,4 \%)$ combinaban la lengua española con otros idiomas. De estas 159 ofertas, $136(65,3 \%)$ incluyeron por lo menos una palabra en inglés y $23(11,1 \%)$ por lo menos una palabra de otro idioma. Lo que significa que más tres cuartos de las ofertas de empleo analizadas $(87,5 \%)$ contenía por lo menos una palabra inglesa como por ejemplo: manager, buman resource, marketing, management, business, outsourcing, service, engineer, enterprise y expert. Mediante una prueba binomial para una única muestra, se ha podido constatar que hay una diferencia estadísticamente significante $(p=.000)$ entre el porcentaje de ofertas que contenían por lo menos una palabra inglesa y el porcentaje de ofertas escritas exclusivamente en español. En otras palabras, la proporción de anuncios con inglés de la muestra se diferencia significativamente del valor hipotético del $50 \%$.

El promedio de la cantidad de palabras inglesas usadas en las ofertas de empleo escritas parcialmente en inglés era de 96 palabras (mínimo una palabra, máximo 540 palabras, $D T=147$ ). Estos anuncios contenían un promedio de 269 palabras (mínimo 59, máximo 1.089, $D T=163)$. Y el porcentaje medio de palabras inglesas aparecidas en estas ofertas fue de $31,65 \%$ con una desviación típica de 41,7. Además, hemos podido constatar una correlación significativa y positiva entre el número de palabras inglesas 
y el número total del palabras en las ofertas redactadas parcialmente en español $\left(\mathrm{r}_{\mathrm{s}}=.427, p<.01, n=136\right)$. Es decir, que a mayor cantidad de palabras usadas para la redacción de un anuncio dado, mayor es el número de palabras inglesas usadas en ese mismo anuncio.

\subsection{Presencia del inglés según el sector de actividad económica}

La mayoría (91 ofertas, 67\%) de las 136 ofertas de empleo escritas parcialmente en inglés se concentran en los siguientes seis sectores de actividad tal y como puede observarse en la Tabla 1: en primer lugar el sector de la 'información y comunicación', las TIC; en segundo lugar las 'actividades administrativas y servicios auxiliares' por ejemplo, actividades relacionadas con el alquiler de maquinaria o vehículos, agencias de colocación; en tercer lugar las 'actividades profesionales, científicas y técnicas' por ejemplo, actividades relacionadas con la contabilidad y servicios técnicos de arquitectura e ingeniería; y finalmente y compartiendo un cuarto lugar los sectores de 'actividades financieras y de seguros', el 'comercio detallista y al por mayor' y el sector de la hostelería.

Tabla 1. Clasificación de los sectores con mayor concentración de inglés según la cantidad de ofertas que contienen inglés.

\begin{tabular}{|l|c|}
\hline Sector de actividad económica & Cantidad de ofertas \\
\hline 1. Información y comunicación & $38(27,9 \%)$ \\
\hline 2. Actividades administrativas y servicios auxiliares & $12(8,8 \%)$ \\
\hline 3. Actividades financieras y de seguros & $11(8,1 \%)$ \\
\hline 4. Información y comunicación & $10(7,4 \%)$ \\
\hline 4. Hostelería & $10(7,4 \%)$ \\
\hline 4. Comercio detallista y al por mayor & $10(7,4 \%)$ \\
\hline & $91(67 \%)$ \\
\hline
\end{tabular}

Atendiendo a la presencia del inglés en las ofertas de empleo de los seis sectores antes mencionados (véase la Tabla 2 para los datos en detalle), el sector que presenta un mayor número de palabras inglesas es el de la 'información y comunicación'; seguido en segundo lugar por el sector de 'actividades profesionales, científicas y técnicas'; en tercer lugar aparece el sector de 'actividades administrativas y servicios auxiliares'; en cuarto lugar el 'comercio al por mayor y al por menor'; en quinto lugar el sector de la 'hostelería'; finalmente y ocupando el sexto lugar encontramos el sector de 'actividades financieras y de seguros'. 
Tabla 2. Clasificación de los sectores según la cantidad de palabras en inglés.

\begin{tabular}{|l|c|c|c|}
\hline Sector & $\begin{array}{l}\text { Cantidad } \\
\text { de ofertas }\end{array}$ & $\begin{array}{l}\text { Cantidad media } \\
\text { de palabras }\end{array}$ & $\begin{array}{l}\text { Desviación } \\
\text { típica }\end{array}$ \\
\hline 1. Información y comunicación & 38 & 143,6 & 165,7 \\
\hline 2. Actividades profesionales, científicas y técnicas & 11 & 104,6 & 160,2 \\
\hline 3. Actividades administrativas y servicios auxiliares & 12 & 97,6 & 133,4 \\
\hline 4. Comercio detallista y al por mayor & 10 & 83,4 & 170,3 \\
\hline 5. Hostelería & 10 & 55,5 & 81,6 \\
\hline 6. Actividades financieras y de seguros & 10 & 5,4 & 3,1 \\
\hline Total & 91 & 81,68 & 119,65 \\
\hline
\end{tabular}

La prueba estadística de Kruskal-Wallis mostró que existe una diferencia significativa de la mediana de palabras inglesas usadas a través de los seis sectores $(H(5)=14.625, p<.05, \mathrm{n}=91)$. Para localizar qué sectores se diferenciaban, hemos realizado la prueba escalonada de comparación por pares de la $U$ Mann-Whitney. De esta serie de comparaciones solo se constató que la mediana de palabras inglesas usadas en el sector de la ‘información y comunicación' $(M e=47.00)$ era significativamente más alta $(U=110.5, z=-2.021, p<.05)$ que en el resto de sectores, a saber, el sector de actividades 'financieras y de seguros' $(M e=5,00)$, 'comercio al por mayor y al por menor' $(M e=5,50)$, 'hostelería' $(M e=6,50)$, 'actividades administrativas y servicios auxiliares' $(M e=17,50)$, actividades profesionales, científicas y técnicas’ $(M e=25)$.

\subsection{Presencia del inglés según el rango de la vacante}

Según la clasificación de ocupaciones laborales por rangos establecida por la OIT (Organización Internacional del trabajo, ver Hoffmann \& Scott, 2011), las 157 ofertas que contenían por lo menos un anglicismo o habían sido redactadas parcialmente o totalmente en inglés han quedado distribuidas de la siguiente manera: 121 ofertas $(77,1 \%)$ para cargos de rango alto, 25 ofertas $(15,9 \%)$ para cargos de rango intermedio y $11(7,0 \%)$ rango bajo.

La aplicación de la prueba estadística de Kruskal-Wallis ha mostrado que hay una diferencia estadísticamente significativa entre el número de palabras inglesas y el rango de la función del cargo $(\mathrm{H}(2)=7.264, \mathrm{p}=.026)$. Según un análisis por pares realizado con la prueba de la $U$ de Mann-Whitney, con una corrección Bonferroni $(p=.017)$, la mediana de palabras inglesas usadas en las ofertas de empleo para profesiones de rango alto $(M e=26, n=121)$ era significativamente mayor que la mediana de las ofertas para las profesiones de rango medio $(M e=8, n=21), U=110.5, z=-2.021, p<.05, r=.20$. No obstante, no se observaron diferencias significativas entre las medianas del grupo de anuncios de empleo para funciones de rango elemental y medio, $U=95.5, z=-.402, p$ $=.688$, como tampoco entre los anuncios para funciones de rango elemental y alto, $U=633.5, z=-.264 p=.792$. 
La relación entre la cantidad de palabras inglesas usadas en las ofertas de empleo y el rango de la vacante ha sido investigada usando el coeficiente de correlación Spearman. Se ha localizado una relación positiva y estadísticamente significativa, aunque no muy fuerte, entre ambas variables $\left(\mathrm{r}_{\mathrm{s}}=.180, \mathrm{n}=136, p<.05\right)$, de tal modo que cuanto mayor es la cantidad de palabras inglesas usadas en las ofertas más alto es el rango de la vacante ofrecida en dichas ofertas de empleo.

\subsection{Presencia del inglés según el tipo de organización sea internacional o nacional}

Según demuestra la prueba de la $U$ de Mann-Whitney, en los anuncios de empleo de las empresas internacionales la mediana de palabras inglesas usadas $(M e=232, n=$ 138) era significativamente mayor que la mediana de palabras inglesas usadas $(M e=$ 201, $n=39)$ en las ofertas de empleo de las empresas nacionales, $U=2140, z=-1.959$, $p=.05, r=.01$.

\section{DISCUSIÓN Y CONCLUSIÓN}

El objetivo de la investigación ha sido cuantificar la presencia del inglés en las ofertas españolas de Monster.es y comprobar hasta qué punto la presencia de esta lengua depende del sector económico de la oferta de empleo, el rango de la función de la vacante y el tipo de empresa.

La presencia de palabras inglesas en el corpus estudiado aquí se ha cifrado en un $39,8 \%$ (PI 1). Este porcentaje supera con creces los porcentajes fijados por Quilis (1984) para el discurso coloquial, los porcentajes fijados por Mighetto $(1991,1992)$ y Rodríguez González (2002a) para la prensa española a partir del Banco de Datos de Prensa Española del año 1977 y el porcentaje fijado por van Hooft (2006), para los anuncios aparecidos en la revista Elle. E incluso supera el porcentaje del 15,22\% más reciente localizado por Gerding et al. (2012) en su estudio sobre el préstamo en la prensa en seis geolectos de la lengua española.

Una gran mayoría, un 87,5\%, de las ofertas de empleo han incluido una o más palabras en inglés. Podemos concluir que el porcentaje de ofertas que contienen inglés supera significativamente el porcentaje de ofertas escritas exclusivamente en español (PI 2). Los resultados obtenidos aquí son muy similares a los obtenidos por van Meurs et al. (2006), en el corpus de ofertas de empleo publicadas en el 2004 en el portal neerlandés www.monsterboard.nl. Lo cual podría indicar que el medio, Internet, y el género textual, las ofertas de empleo, serían dos factores determinantes que explicarían esta presencia tan masiva de préstamos no adaptados del inglés, independientemente de la lengua que los adopta.

Según los porcentajes obtenidos en los estudios arriba citados y desde una perspectiva diacrónica y comparación multimodal y a través de diferentes géneros orales y escritos (ver los estudios presentados en la introducción), podría establecerse 
que, por un lado, la presencia de préstamos no adaptados procedentes inglés en la lengua española ha aumentado a lo largo del periodo 1984 y 2012, por lo menos en los corpus estudiados. Por otro lado, y si se comparan los porcentajes de casos de palabras inglesas aparecidas tanto en la prensa como la publicidad en español y el porcentaje de casos localizados en el corpus aquí estudiado, los datos parecen indicar que, a pesar de las diferencias de tamaño entre los diferentes corpus y el tiempo en que han sido recogidos, el medio y el género textual podrían también, como indicábamos en el párrafo anterior, ser factores que determinen el grado de influencia del inglés en la lengua española. Para comprobar la validez de esta hipótesis, sería necesario generar más datos procedentes de diversos corpus y contrastarlos.

La presencia masiva del inglés en el corpus estudiado podría explicarse, en primer lugar, por la naturaleza del medio, Internet, un espacio relativamente nuevo, abierto, internacional, interactivo, flexible y muy permeable a las modas y tendencias dentro de nuestras sociedades hispanas y del resto del mundo. En segundo lugar, la presencia del inglés vendría además motivada por la interdependencia de Internet con las TIC, sector este que precisamente es el que registra un mayor número de anglicismos no adaptados comparado con el resto de sectores de actividad económica.

En tercer lugar, el hecho de que muchas empresas internacionales y multinacionales están presentes en muchos de países de habla hispana sería otro factor que explicaría que, precisamente, en este género textual hayamos observado una mayor presencia del inglés.

Nuestro análisis ha mostrado que la cantidad de palabra usadas en las ofertas de empleo depende del sector económico que ocupa la empresa que coloca el anuncio (PI 3). Este dato apoya los resultados obtenidos por van Meurs et al. (2006) en el caso neerlandés y, a su vez, las expectativas indicadas por otros estudios dedicados a la publicidad y prensa en español (por ejemplo, Blanco, 1995; Lorenzo, 1996; Calvi, 2000; Lázaro Carreter, 2002; Rodríguez González 2002a; Álvarez, 2003; van Hooft, 2006; Cruz et al., 2007; Gerding et al., 2012) según los cuales el uso del inglés en la lengua española está ligado a determinados sectores o ámbitos profesionales, sociales y culturales. En nuestro estudio, el sector de las 'tecnologías de la información y comunicación' es el que muestra una mayor presencia significativa de anglicismos no adaptados en comparación con el resto de sectores. Este hecho concuerda también con los resultados obtenidos en el estudio de van Meurs et al. (2006) dedicado a las ofertas de empleo neerlandesas publicadas en www.monster.nl. Además el hecho de que las TIC encabecen el índice de adopción de anglicismos no solo en los dos estudios dedicados a las ofertas de empleo, pero también en otros ámbitos como el de la prensa escrita en español tal y como han constatado Gerding et al. (2012), parece indicar que no es una casualidad que, precisamente, este sector sea el más permeable a la influencia del inglés en el ámbito de habla hispana, por lo menos en los géneros estudiados hasta hoy. 
Según los resultados obtenidos en nuestro estudio, la presencia de anglicismos en el corpus estudiado depende significativamente del rango de la vacante anunciada en las ofertas de empleo (PI 4) de tal modo que las ofertas de empleo para ocupaciones de rango alto usan más anglicismos que las ofertas para ocupaciones medias y bajas. Nuestros resultados coinciden con los obtenidos por van Meurs et al. (2006). A pesar de que la correlación positiva constatada entre el rango de la vacante y la cantidad de palabras inglesas se dé en dos lenguas pertenecientes a dos familias lingüísticas distintas, no significa que, obviamente, la conclusión sea generalizable. Para ello es indispensable recabar más evidencias en otros países de habla hispana y otros ámbitos lingüísticos.

Un argumento que podría explicar que el inglés aparezca con más frecuencia en las ocupaciones de rango alto es la posible asociación de la función ofertada al prestigio social del inglés y la función del inglés como lengua franca en, por ejemplo, áreas de actividad humana como el comercio internacional, las finanza y la enseñanza terciaria (Harmann, 1989; Takahashi, 1990; Gerritsen et al., 2000). Los resultados obtenidos en nuestro análisis apoyarían esta explicación dado que las 23 ofertas escritas íntegramente en inglés, anunciaban, todas ellas, vacantes de rango alto de empresas internacionales y multinacionales. Por otro lado, las ofertas vacantes para cubrir cargos o funciones altas redactadas exclusivamente en inglés, estarían dirigidas a un grupo objetivo con un perfil profesional muy determinado que por lo menos incluya un nivel alto de educación y un muy buen dominio del inglés como lengua extranjera (Piller, 2003). Además, y para las ofertas de empleo redactadas exclusivamente en inglés, la elección de esta lengua tendría como finalidad preseleccionar a los candidatos, ya que todas aquellas personas hispanohablantes que no puedan leer y entender el mensaje escrito en inglés quedan excluidas a priori del proceso de selección para cubrir la plaza vacante.

Nuestro análisis de corpus muestra que la presencia de anglicismos depende del carácter internacional de la empresa (PI 5), puesto que las empresas internacionales y multinacionales contenían significativamente más anglicismos que las ofertas colocadas por empresas nacionales. Los resultados obtenidos apuntan en la misma dirección que los resultados de Korzilius et al. (2006) y van Meurs et al. (2006). Resultados que, su vez, sustentan las expectativas de Larson (1990), Gerritsen et al. (2000) y Alm (2003), según las cuales las empresas internacionales y multinacionales usarían la lengua inglesa para crear una imagen internacional consistente. Confirmando de esta manera que dichas empresas han elegido una misma estrategia de marketing global donde el inglés es la lengua de comunicación entre la empresa y los posibles candidatos a ocupar posiciones en dichas empresas, todos ellos personas pertenecientes a distintos países y áreas lingüísticas (Jain, 1989; De Mooij, 1994; Piller, 2001).

Lo dicho hasta ahora nos permite concluir que los factores estudiados en este corpus determinan la elección lingüística, el impacto y la presencia del inglés en 
las ofertas de empleo publicadas en www.monster.es. Luego la presencia del inglés observada en nuestro estudio, vendría motivada no solo por la función simbólica del inglés pero además y quizás principalmente, por razones prácticas y funcionales dado que el inglés se usa en ofertas de empresas de clara orientación internacional, para ofertar vacantes de alto nivel, y en el sector de las TIC donde el inglés suele ser lengua franca. Los resultados obtenidos constatan la dinámica y las influencias entre hablantes de diferentes lenguas que crean espacios multilingües, ricos y complejos en los que, en el caso estudiado, concurren el español como lengua nativa y el inglés como lengua extranjera. Y por último, los datos obtenidos pueden considerarse como un indicador de la expansión de las áreas de influencia del inglés y, a la vez, como un síntoma de la globalización.

Este trabajo tiene una serie de limitaciones como, por ejemplo, el tamaño relativamente pequeño de la muestra estudiada, el hecho de que no hayamos tenido en cuenta en qué partes de las ofertas de empleo aparecen más o menos anglicismos. O que solo hayamos partido en nuestros análisis de los casos y no hayamos tenido en cuenta los tipos de 'préstamos no adaptados' procedentes del inglés. Tampoco hemos distinguido en nuestro análisis la distribución de los anglicismos según categorías gramaticales, o presentado las listas de los anglicismos aparecidos y organizarlos, por ejemplo, en campos semánticos o sospesar la relevancia de los mismos. Además, solo hemos investigado el sitio de www.monster.es en España. De ahí que para obtener un mapa más amplio y detallado, y comprobar si ocurre algo parecido en el resto de países de habla hispana, sea necesario replicar este tipo de investigaciones. De esta manera podremos calibrar y entender mejor la influencia del inglés en la lengua española. 


\section{REFERENCIAS BIOBLIOGRÁFICAS}

Alm, C. (2003). English in the ecuadorian commercial context. World Englishes, 22(2), 143-158.

Álvarez, I. (2011). El ciberespañol: Características del español usado en Internet. En L. Ortiz-López (Ed.), Selected Proceedings of the 13th Hispanic Linguistics Symposium (pp. 33-41). Somerville, MA: Cascadilla Proceedings Project.

Baumgardner, R. J. (2006). The appeal of English in Mexican commerce. World Englishes, 25(2), 251-266.

Bhatia, T. (2001). Language mixing in global advertising. En E. Thumboo (Ed.), The Three Circles of English (pp. 195-216). Singapur: University Press.

Blanco, L. (1995). La neología: Algunos usos en el español actual. Español Actual, 64, 27-38.

British National Corpus (2011). Oxford: Universidad de Oxford [en línea]. Disponible en: http://www.natcorp.ox.ac.uk/using/index.xml

Buades, A. \& González, F. (2009). Nuevo diccionario de anglicismos. Madrid: Gredos.

Calvi, M. V. (2000). Il linguaggio spagnolo del turismo. Viareggio-Lucca: Mauro Baroni Editore.

CBS (Centraal Bureau voor de Statistiek) (2010). Standaard beroepenclassificatie [en línea]. Disponible en: http://www.cbs.nl/NR/rdonlyres/6221F84D-BEDD-4B0CB9ED-ADF076E4D769/0/2010sbcclassificatieschema.pdf

Cheshire, J. \& Moser, L. (1994). English as a cultural symbol: The case of advertisements in French-speaking Switzerland. Journal of Multilingual and Multicultural Development, 15, 451-469.

Comisión Europea (2006). Statistical Classification of Economic Activities in the European Community, NACE Rev. 2 [en línea]. Disponible en: http:// ec.europa.eu/eurostat/ramon/nomenclatures/index.cfm?TargetUrl=LST_ NOM_DTL\&StrNom $=$ NACE_REV $2 \& S \operatorname{trLanguageCode}=$ EN\&IntPcKey $=\&$ StrLayoutCode $=$ HIERARCHIC

Crystal, D. (2003a). English as a global language. Cambridge: University Press.

Crystal, D. (2003b). The Cambridge Encyclopaedia of the English Language, Cambridge: Cambridge University Press.

Cruz, I., Tejedor, C., Díez, M. \& Cerdá, E. (2007). English loanwords in Spanish computer language. English for Specific Purposes, 26, 52-78. 
Durán Martínez, R. (2000). Análisis estadístico de la presencia de la lengua inglesa en la publicidad comercial española. Aula, 12, 87-101.

De Mooij, M. (1994). Advertising worldwide: Concepts, theories and practice of international, multinational and global advertising. Nueva York: Prentice Hall.

Friedrich, P. (2002). English in advertising and brand naming: Sociolinguistic considerations and the case of Brazil. English Today, 18(3), 21-28.

Gerding, C., Fuentes, M., Gómez, L. \& Kotz, G. (2012). El préstamo en seis variedades geolectales del español: Un estudio en prensa escrita. Revista Signos. Estudios de Lingüistica , 45(80), 280-299.

Gerritsen, M., Korzilius, H., van Meurs, F. \& Gijsbers, I. (2000). English in dutch commercials: Not understood and not appreciated. Journal of Advertising Research, 40(3), 17-31.

Gerritsen, M., Nickerson, C., Crijns, R., van Hooft, A., van Meurs, F., Nederstigt, U. $\&$ Starren, M. (2007). English in product advertisements in Belgium, France, Germany, the Netherlands and Spain. World Englishes, 26(3), 291-315.

Griffin, J. (1997). Global English invades Poland. An analysis of the use of English in Polish Magazine Advertisements, English Today, 13(2), 34-41.

Gómez Capuz, J. (2009). La asimilación gramatical de los anglicismos en un corpus de español coloquial (II): Formación del plural de los sustantivos y asimilación de adjetivos y verbos. Lingüistica Española Actual, 31(2), 299-232.

Google (2011) [en línea]. Disponible en: https://www.google.co.uk/

Ngram Viewer (2011) [en línea]. Disponible en: https://books.google.com/ngrams

Görlach, M. (Ed.) (2002). English in Europe. Oxford: Oxford University Press.

Haarmann, H. (1989). Symbolic values of foreign language use: From the Japanese Case to a General Sociolinguistic Perspective. Berlín: Mouton de Gruyter.

Hilgendorf, S. K. (1996). The impact of English in Germany. English Today, 12(3), 3-14.

Hoffmann, E., Scott, M. \& Organización Internacional del Trabajo (2011). The Revised International Standard Classification of Occupation (ISCO-88). A Short Presentation [en línea]. Disponible en: http://www.ilo.org/public/english/bureau/stat/ download/papers/short.pdf

IBM Corp. (2012). IBM SPSS Statistics for Windows, Version 21.0. Armonk, Nueva York: IBM Corp.

INE (Instituto Nacional de Estadística) (2011). Clasificación de Ocupaciones [en línea]. Disponible en: http://www.ine.es/daco/daco42/discapa/tar1.pdf 
Jain, S.C. (1989). Standardization of international marketing strategy: Some research hypotheses. Journal of Marketing, 53, 70-79.

Jain, S.C. (1993). International marketing management. Belmonte: Wadsworth Publishing Company.

Kelly-Holmes, H. (2000). Bier, Parfum, Kaas. Language fetish in European advertising. European Journal of Cultural Studies, 3, 67-82.

Korzilius, H. (2000). De Kern van Survey-onderzoek. Assen: Van Gorcum.

Korzilius, H, van Meurs, F. \& Hermans, J. (2006). The use of English in job advertisements in a Dutch national newspaper: On what factors does it depend? En R. Crijns \& C. Burgers (Eds.), Werbestrategien in Theorie und Praxis: Synchrone, diachrone und interkulturelle Perspektive (pp. 147-174). Tostedt: Attikon Verlag.

Larson, B. E. (1990). Present-day influence of English on Swedish as found in Swedish job advertisements. World Englishes, 9, 367-369.

Lázaro Carreter, F. (2002). El neologismo en el DR AE. Academia 15 [en línea]. Disponible en: http://www.rae.es/rae/gestores/gespub000001.nsf/(voAnexos)/arch5779 15B193FE3862C1256B6800363ED2/\$FILE/neologismoflc.htm

Lorenzo, E. (1996). Anglicismos hispánicos. Madrid: Gredos.

Mighetto, D. (1991). Las palabras-cita y los libros de estilo. Moderna Språk, 82(2), 180185.

Mighetto, D. (1992). Palabras-cita en la prensa española. En R. Lorenzo (Ed.), Actas do XIX Congreso Internacional de Lingüistica e Filoloxia Románicas (pp. 564-584). A Coruña: Universidad de Santiago de Compostela / Fundación Pedro Barrié de la Maza, Conde de Fenosa.

Merriam-Webster Online Dictionary (2010) [en línea]. Disponible en: http:// www.m-w.com/

Oxford Online Dictionary (2010) [en línea]. Disponible en: http://oxforddictionaries. $\mathrm{com} /$

Piller, I. (2001). Identity constructions in multilingual advertising. Language in Society, 30, 153-186.

Piller, I. (2003). Advertising as a site of language contact. Annual Review of Applied Linguistics, 23, 170-183. 
Quilis, A. (1984). Anglicismos en el español de Madrid. En L. A. de Cuenca, E. G. Elícegui, A. B. Pajares \& J. L. Facal (Coord.), Athlon: Satura grammatica in honorem Francisci R. Adrados (pp. 413-422). Madrid: Editorial Gredos.

Real Academia Española (2003). Diccionario de la Real Academia Española [DRAE]. [CDROM]. Versión 1.0. Madrid: Espasa Calpe.

Real Academia Española (2003). Diccionario de la Real Academia Española [DRAE] [en línea]. Disponible en: http://www.rae.es/recursos/diccionarios/drae

Rodríguez González, F. (2002a). Spanish. En M. Görlach (Ed.), English in Europe (pp. 1528-150). Oxford: Oxford University Press.

Rodríguez González, F. (2002b). Spanish. En M. Görlach (Ed.), An Annotated Bibliography of European Anglicisms (pp. 228-247). Oxford: Oxford University Press.

Taavitsainen, I. \& Pahta, P. (2003). English in Finland: Globalisation, language awareness and questions of identity. English Today, 19(4), 3-15.

Takahashi, K. (1990). A sociolinguistic analysis of English borrowings in Japanese advertising texts. World Englishes, 9(3), 327-341.

Ustinova, I.P. (2006). English and eemerging advertising in Russia. World Englishes, 25(2), 267-77.

van der Sijs, N. (1996). Leenwoordenboek: De invloed van andere talen op het Nederlands. Den Haag: SDU.

van Hooft, A. (2006). El valor añadido de los anglicismos en la publicidad española. El impacto y la valoración social de las voces inglesas en los anuncios de la Revista Elle. En M.V. Calvi \& L. Chierichetti, (Eds.), Nuevas Tendencias en el Discurso de Especialidad Linguistics Insights. Studies in Language and Communication (pp. 219-243). Frankfurt am Main: Peter Lang.

van Meurs, F., Korzilius, H. \& Hollander, A. (2006). The Use of English on the Dutch Job Site Monsterboard.nl and factors on which it depends. ESP Across Cultures, 3, 102-123.

Walsh, L.S. (1991). International marketing, Londres: Financial Times Pitman.

Zenner, E., Speelman, D. \& Geeraerts, D. (2013). Macro and micro perspectives on the distribution of English in Dutch. A quantitative usage-based analysis of job ads. Linguistics, 51(5), 1019-1064.

\section{* AGRADECIMIENTOS}

El análisis del corpus ha sido posible gracias a la colaboración de Cassy Smith y Kirsten Hendrikx. 